\title{
Infrared Radiation From a Bunsen Flame
}

\author{
By Earle K. Plyler
}

\begin{abstract}
The infrared emission spectrum of the flame of a Bunsen burner has been measured with prisms of $\mathrm{LiF}, \mathrm{NaCl}$, and $\mathrm{KBr}$. In the region of $2.7 \mu$, the emission band has been resolved into two bands, 2.5 and $2.7 \mu$, and a series of 15 lines from 2.8 to $3.1 \mu$. These emission lines are separated by about $22 \mathrm{~cm}^{-1}$ and fall in the spectral region where the rotational-vibrational band of $\mathrm{OH}$ should occur. The spacing between lines, however, is too small for the changes in the energy levels of the $\mathrm{OH}$ molecule. This separation agrees very well with the calculated value for certain rotational states of the $\mathrm{H}_{2} \mathrm{O}$ molecule. The long wavelengih band at $15 \mu$ has been observed in both CO burned in the air and the Bunsen burner flame. The present detection of this band represents the first confirmation of the original observations of Rubens and Aschkinass. Several bands have been observed with a grating instrument, and their frequencies check well with known energy transitions. The region from 12 to $24 \mu$ has been measured with a $\mathrm{KBr}$ prism for the Bunsen flame. It contains a large number of the pure rotational lines of water vapor. In addition to the $\mathrm{H}_{2} \mathrm{O}$ lines, there are two strong bands, 14 and $15 \mu$, which are produced by $\mathrm{CO}_{2}$.
\end{abstract}

\section{Introduction}

Many observers have studied the infrared emission of a Bunsen flame [1]. ${ }^{2}$ The strongest band occurs at about $4.4 \mu$ and is produced by carbon dioxide. Other bands have been found at 0.95 , $1.45,2.0$, and $2.8 \mu$, and are attributed to carbon dioxide and water vapor. Very little radiation has been observed in the region beyond $7 \mu$.

In the visible and ultraviolet regions of the spectrum, considerable progress has been made in the study of flames [2]. By the use of instruments with good resolution and by detecting with photographic plates that average out the fluctuations of the flame, many band systems have been identified. The band systems include such atomic groups as $\mathrm{OH}, \mathrm{CN}, \mathrm{NO}, \mathrm{CH}$, and $\mathrm{NH}$.

The question may be asked why the spectra of such groups have not been observed in the infrared region. The probable reason is that sufficiently high resolution has not been applied in the infrared region to separate properly the spectra of the different groups. Also, slight fluctuations in the flames make it difficult to obtain accurate meas-

\footnotetext{
${ }_{1}^{1}$ Part of the subject matter of this paper was presented at the Cincinnati meeting of the Optical Society of America on October 23 to 25, 1947.

${ }_{2}^{2}$ Figures in brackets indicate the literature references at the end of this paper.
}

urements with radiometric detectors. An advantage in the study of the infrared spectra of flames is that a large percentage of the energy emitted by the flame falls in this region. The present study is mainly confined to the flame of the Bunsen burner. Both natural and mixed manufactured-natural gases were used.

\section{Experimental Method and Results}

A Perkin-Elmer infrared spectrometer was used in the measurement of the spectra, with prisms of $\mathrm{LiF}, \mathrm{NaCl}$ and $\mathrm{KBr}$ for the different regions of the spectrum. A Bunsen burner with a steady flame was substituted for the globar source. Observations were made on the flame in the region of the cone, about two thirds of the distance from the bottom of the cone. Although the flame appeared steady, there was some variation in the total radiation that may have been produced by small fluctuations in the gas pressure. In many cases, a region was observed five or six times, and an average curve calculated from the various observations. The spectra shown have been averaged. As a rule, a single trial would not yield such a smooth curve. However, no emission band was included unless it could be redetermined several times. For this 
reason, there may be more structure in the bands than is actually shown.

Figure 1 shows the energy curve for the Bunsen burner as obtained with a $\mathrm{NaCl}$ prism in the region from 1 to $5 \mu$. The lowest curve was observed with slits $60 \mu$ wide, and the slits were $100 \mu$ wide for the upper curve from 1.5 to $4.0 \mu$. In the region from 1 to $1.5 \mu, 200-\mu$ slits were necessary to observe the bands. In the region of

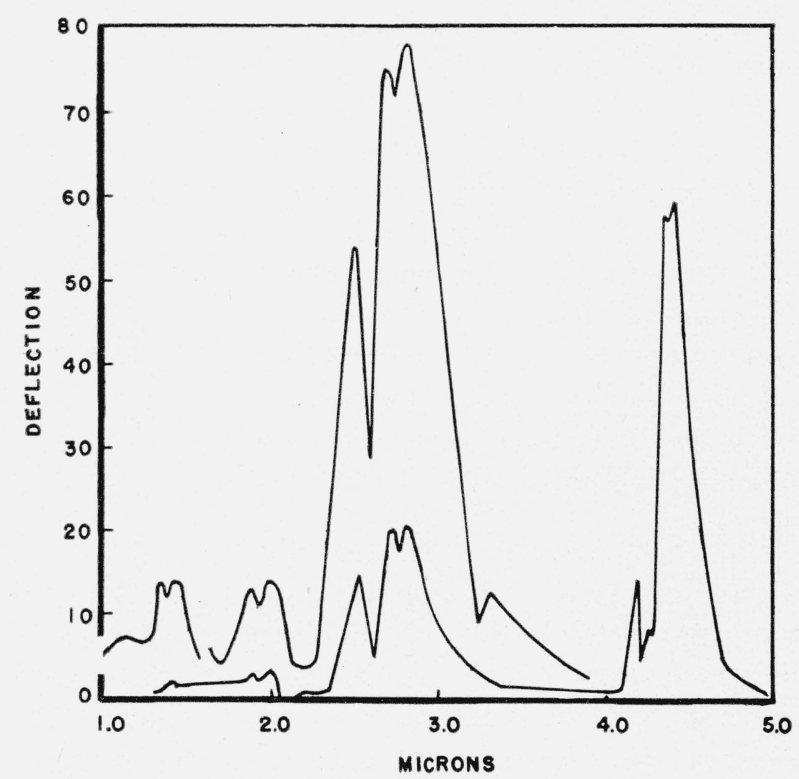

FigurE 1. Infrared emission of a Bunsen flame measured with a $\mathrm{NaCl}$ prism.

The upper curve was measured with wider slits than the lower.
$4.25 \mu$, there is a drop in the energy curve produced by the absorption of the carbon dioxide in the air. The strong band at $4.39 \mu$ is somewhat irregular at the point of greatest energy and can be observed better under higher resolution.

The region from 1.7 to $3.6 \mu$ has been observed under higher resolution by the use of a LiF prism. The results are shown in figure 2 . The slits used wére $60 \mu$ wide.

These results were obtained with natural gas containing about 88 percent of methane. The small bands in the region of 1.8 to $2 \mu$ are produced by water vapor and $\mathrm{CO}_{2}$. The band at $2.49 \mu$ is probably due to water vapor, and the bands in the region of $2.7 \mu$ are attributed to $\mathrm{CO}_{2}$. The fine structure in the region from 2.8 to $3.1 \mu$ may be produced by $\mathrm{H}_{2} \mathrm{O}$. This fine structure extends over to the $2.7-\mu$ region, but because of the absorption of water vapor in the air and the band at $2.79 \mu$ in the LiF prism, it could not be observed with sufficient accuracy to be included. Hence, a smooth curve was drawn thru the observed points in the 2.7- to $2.8-\mu$ region. A small band observed in the region of $3.32 \mu$ is probably produced by $\mathrm{CH}$. This band is of low intensity, and it is not possible to determine whether it arises from $\mathrm{CH}, \mathrm{CH}_{2}, \mathrm{CH}_{3}$ or $\mathrm{CH}_{4}$ It is removed by about $12 \mathrm{~cm}^{-1}$ from the position of the methane absorption band.

In figure 3 is shown the emission band in the region of $4.36 \mu$. The solid line gives the actual

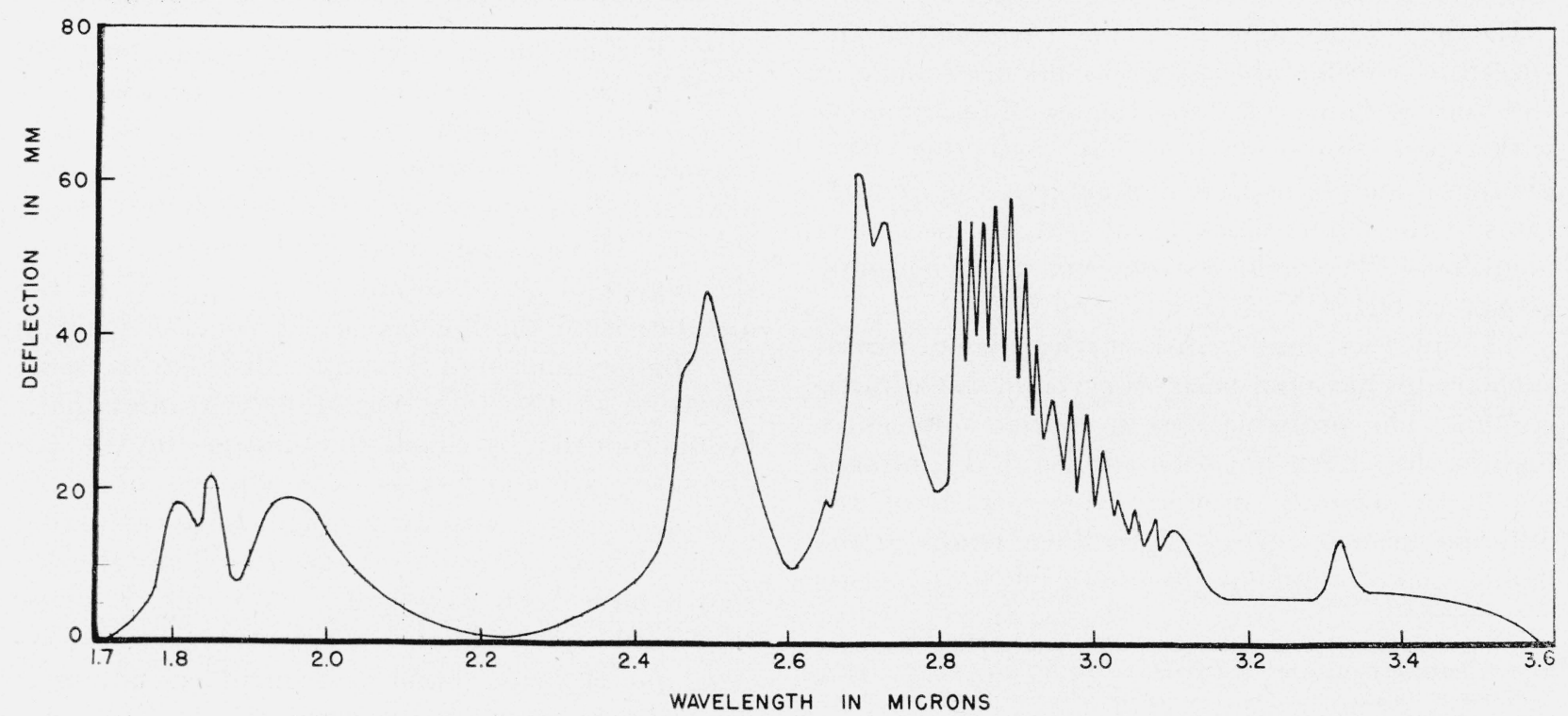

Figune 2. Infrared einission of a Eunsen flame measured with a LiF prism. 
deflections. The reversal of the $\mathrm{CO}_{2}$ absorption band in the air causes the low energy in the region of 4.22 and $4.28 \mu$. The easily recognized doublet band of $\mathrm{CO}_{2}$ can be identified. The broken line in figure 3 indicates the amount of energy that would be present if the $\mathrm{CO}_{2}$ in the air did not absorb any of the radiation emitted by the flame. The corrections to the observed curve were averaged from six observations of the absorption of $\mathrm{CO}_{2}$ in this region. As the flame changes the amount of $\mathrm{CO}_{2}$ in the air, it was found necessary to take a reading of the radiation of the flame and, immediately afterward at the same wavelength, a reading of the radiation from the globar source. This method of measurement was carried out at frequent settings of the wavelength drum, and the resultant curve was obtained. Barker [3] has obtained a curve of this type by the use of a grating spectrometer. The value of the maximum energy was found by Barker to be $4.37 \mu$.

The absorption by the flame of radiation in the region from 4.0 to $4.8 \mu$ is small. The flame of the Bunsen burner was placed in the path of the radiation from the globar source, but in a position where its flame was not in focus and hence its radiation could produce very little effect on the detector. At $4.36 \mu$ where the maximum emission of $\mathrm{CO}_{2}$ occurs, the globar radiation was reduced very little, and no appreciable absorption

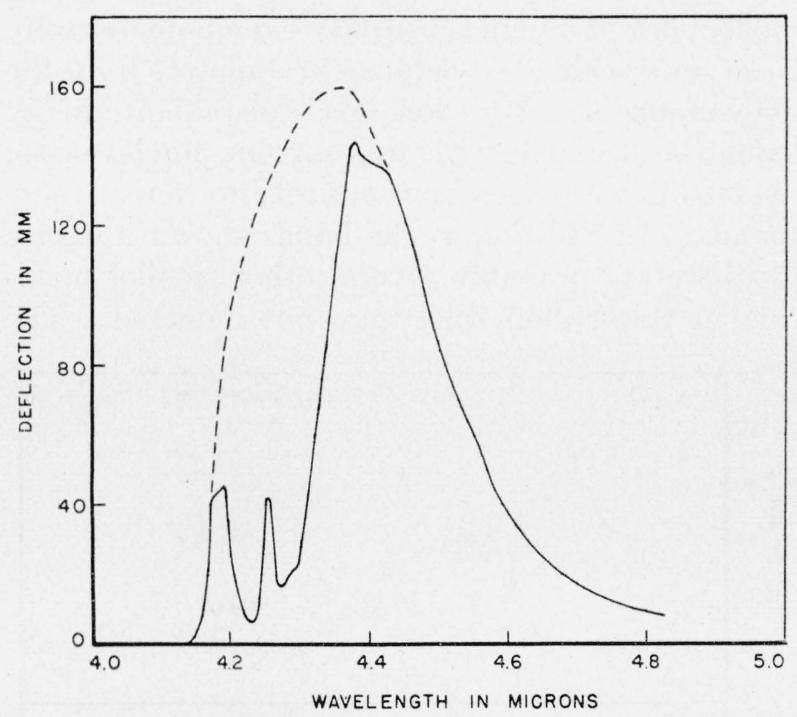

Figure 3. The 4.36- $\mu$ emission band of a Bunsen-burner flame.

The broken line represents the probable form of the energy curve after applying corrections for absorption of $\mathrm{CO}_{2}$ in the air.

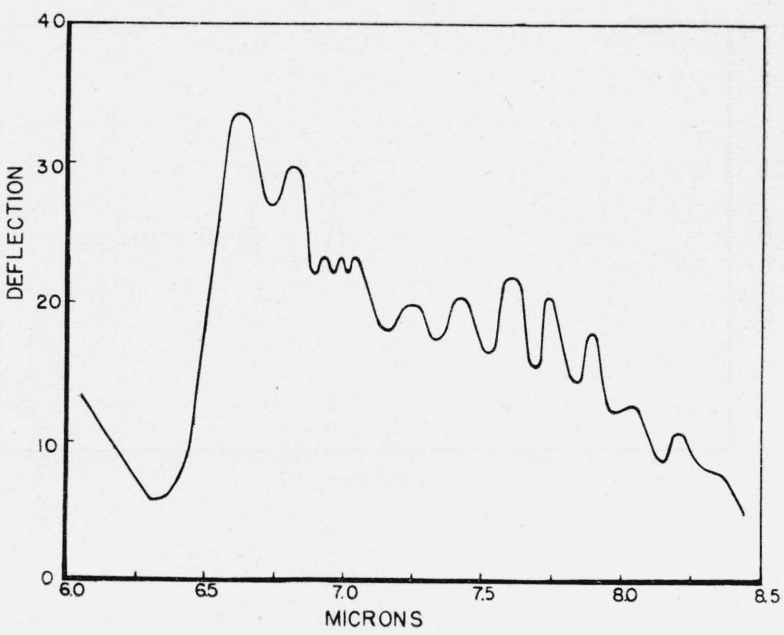

Figure 4. Infrared emission of a Bunsen flame in region 6 io $8.5 \mu$ measured with a $\mathrm{NaCl}$ prism.

due to the flame was found in any part of this spectral region.

In figure 4 is shown the emission spectrum from 6 to $8.5 \mu$. The energy is low in this region so that slits of $300 \mu$ were used. This band is somewhat distorted and is part of the bending vibration of $\mathrm{H}_{2} \mathrm{O}$. In absorption this water-vapor band occurs at $6.26 \mu$. Some lines of the rotationalvibrational band stand out clearly in the region of 7.5 and $8 \mu$. The region from 5 to $6 \mu$ could not be studied in detail because of the overlapping of the strong $\mathrm{CO}_{2}$ band. This band was measured with a $\mathrm{NaCl}$ prism. No doubt, a greater detail of structure could be observed by the use of a $\mathrm{CaF}_{2}$ prism or a grating.

Rubens and Aschkinass [4] in their study of the emission of water vapor, were able to find both branches of this vibrational-rotational band, because they did not have the tremendous overlapping of the $\mathrm{CO}_{2}$ emission band. In the emission curve that they obtained from the Bunsen burner they were able to obtain only the long wavelength portion of the band, as is shown in figure 4 of the present paper. Their instrument was not of sufficient resolution to observe the individual rotational lines. However, their work was remarkably accurate and the bands check well in intensity with the results obtained in the present study.

In figure 5 the rotational lines of water vapor have been observed from 8.5 to $14.5 \mu$ with a $\mathrm{NaCl}$ prism and slits of $0.45-\mathrm{mm}$ width. The energy is very small in this region, and from 9 


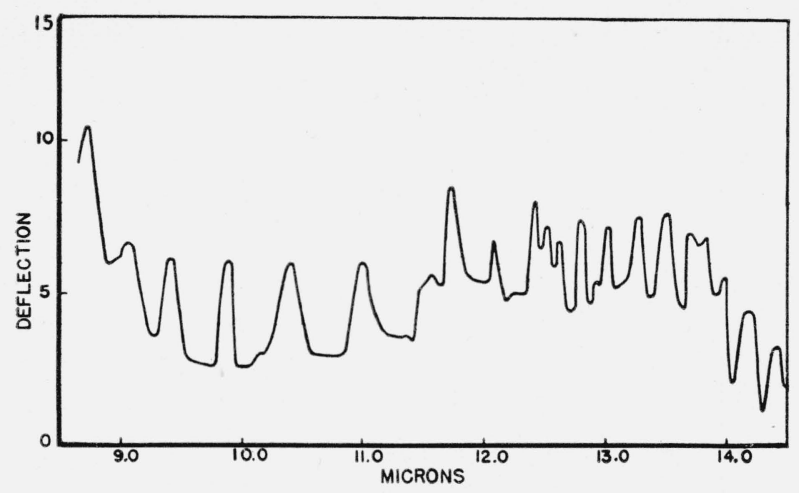

Figure 5. Infrared emission of a Bunsen flame in region 9 to $14 \mu$ measured with a $\mathrm{KBr}$ prism.

to $11 \mu$ it falls to a minimum. This is the part of the spectrum where water vapor has the least absorption, and is also that part of the spectrum where the pure rotational lines of $\mathrm{H}_{2} \mathrm{O}$ begin to appear. The energy increases from 11.5 to 13.5 $\mu$ and then starts to decrease again. The decrease in energy is caused by the absorption of $\mathrm{CO}_{2}$ in the air and the absorption of the prism. In addition to the water-vapor lines in this region, there is a possibility that the two difference bands, $\nu_{3}-2 \nu_{2}$ and $\nu_{3}-\nu_{1}$ are present in low intensities. These bands occur in absorption at 9.40 and $10.41 \mu$. There are two distinct maxima observed in this region, but whether they are produced by $\mathrm{H}_{2} \mathrm{O}$ or $\mathrm{CO}_{2}$ has not been determined because both substances are present in the Bunsen-burner flame. This question can be answered when spectra of burning hydrogen and burning $\mathrm{CO}$ are measured separately in this region. That the decrease in energy is caused by the $\mathrm{CO}_{2}$ and the absorption of the prism is clearly demonstrated when this region is studied with a $\mathrm{KBr}$ prism.

In figure 6 is shown the energy curve from 12 to $24 \mu$. From 12 to $19.5 \mu$ the slits were $0.5 \mathrm{~mm}$ in width; from 19.5 to $21.5 \mu, 0.7 \mathrm{~mm}$; and 21.5 to $24 \mu, 1.0 \mathrm{~mm}$. In contrast with the measurements with the $\mathrm{NaCl}$ prism, which does not give large deflections for this part of the spectrum, the energy observed with the $\mathrm{KBr}$ prism increases from 12 to $15 \mu$. The broken line curve at about $15 \mu$ in figure 6 is a measure of the energy after corrections have been made for the absorption of $\mathrm{CO}_{2}$ in the air. The absorption of $\mathrm{CO}_{2}$ in the air was measured with the same slit width used in the emission measurements, and then corrections to the observed curve were made. The maximum of radiation occurs at $15.0 \mu$. This emission band is no doubt one of the long wavelength $\mathrm{CO}_{2}$ bending vibrations. In the region from 13 to $17 \mu$, there is a considerable number of lines of water vapor, which overlap the $\mathrm{CO}_{2}$ emission. However, the two intense peaks at 13.9 and $15.0 \mu$ are produced by $\mathrm{CO}_{2}$.

In 1944 an unpublished study was made by the author at the University of Michigan on the emission of burning $\mathrm{CO}$, using a grating spectrometer with a $\mathrm{KBr}$ foreprism. The $\mathrm{CO}$ was generated by two different methods. In one case a furnace was used that had a tube in the center filled with charcoal. Oxygen was passed slowly through the heated tube, and the $\mathrm{CO}$ was burned at the end of the tube. In the second case the $\mathrm{CO}$ was generated by dropping formic acid onto heated sulfuric acid. The radiation of the burning $\mathrm{CO}$ was measured, and in each instance, two peaks were found - one at $14.0 \mu$ and the other at $15.0 \mu$. As a further proof that the observed bands were produced by $\mathrm{CO}_{2}$, the charcoal was removed from the tube and $\mathrm{CO}_{2}$ gas was passed slowly through the heated tube. Under these conditions, the radiation in the $15-\mu$ region was also detected.

The results obtained with the grating instrument are shown in figure 7 . The slits of the spectrometer were $1 \mathrm{~mm}$ wide and a 1,200 line per inch grating with an area of 108 in. $^{2}$ was used in the spectrometer. The energy of the thermocouple was amplified by a Moll thermal relay, and the deflections of a high sensitivity galvanometer were read on a scale at a distance of 3 meters from the galvanometer. Very low levels of radiant energy could be detected with this system, but even so, wide slits were necessary in order to detect these bands. In addition to the bands shown in figure 7 , there are probably several other smaller maxima in this region that were not detected. The

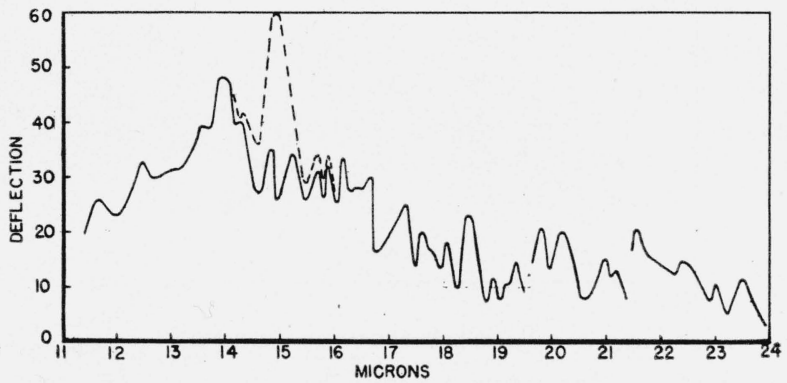

Figure 6. Infrared emission of a Bunsen flame in region 12 to $24 \mu$ measured with a $\mathrm{KBr}$ prism.

The broken line represents the probable form of the energy curve after applying corrections for absorption of $\mathrm{CO}_{2}$ in the air. 


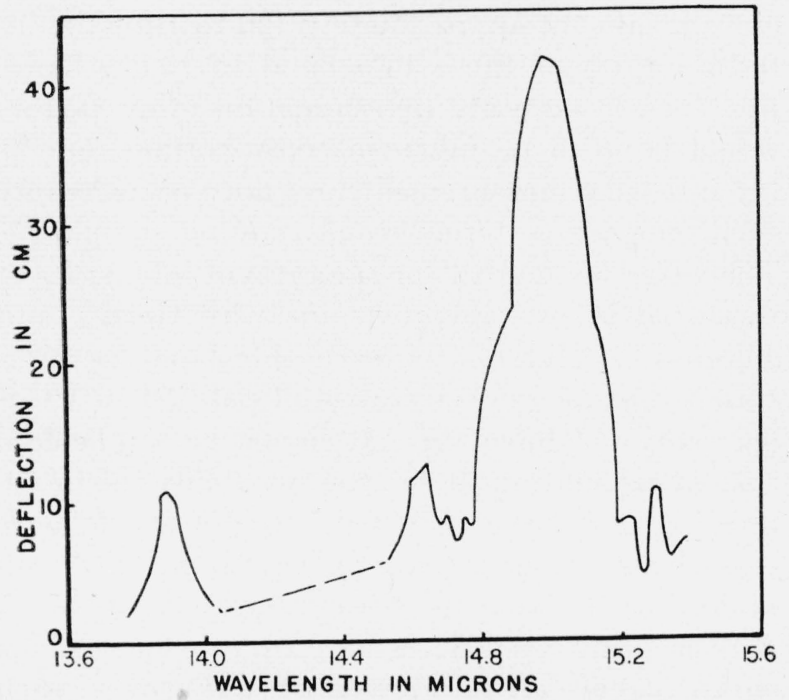

Figure 7. Infrared emission of a $\mathrm{CO}$ flame burning in air measured with a grating spectrometer after applying corrections for absorption of $\mathrm{CO}_{2}$ in the air.

curve represents the probable emission curve after corrections to the deflections were made by taking into consideration the absorption of the $\mathrm{CO}_{2}$ in the air.

Rubens and Aschkinass [4] in 1898 observed a band at $14.1 \mu$, but later observers were not able to find any band in this region. The intensities obtained by Rubens and Aschkinass for the bands of the Bunsen burner flame are in good agreement with results of this study. They measured the emission spectra to $23 \mu$ with a $\mathrm{KCl}$ prism and found a definite peak in the region 13 to $14 \mu$, although their resolution was not great enough to show that this region contained many emission bands and lines. Their success in finding the band was due to the use of the $\mathrm{KCl}$ prism.

Garner and Johnson [5] in 1927 tried to observe this band but were not able to detect any energy in this region. They stated that, if the band was one-fiftieth as strong as the $4.4-\mu$ band, it could be observed. The reasons for the low intensity of this band are discussed in section III.

The emission spectrum of the Bunsen burner from 6.5 to $24 \mu$ has a large number of water vapor lines caused by rotation of the $\mathrm{H}_{2} \mathrm{O}$ molecules. No doubt these lines continue beyond $24 \mu$. The separate emission lines are not quite at the same position in wavelength as the absorption lines. Also there are many other lines that fall between the ones shown in figure 6 , but, because of the low resolution, they could not be observed in sufficient detail for measurement.

\section{Discussion of Results}

The infrared-absorption spectrum of $\mathrm{CO}_{2}$ has been classified by Adel and Dennison [6], and, by means of their equations and certain observed bands, it is possible to calculate the frequency corresponding to the various quantum states. The notation followed in this discussion to designate the different types of vibration of $\mathrm{CO}_{2}$ and $\mathrm{H}_{2} \mathrm{O}$ is that used by Herzberg [7] in the classification of these molecules. For example, the $\nu_{3}$ band, which is the asymmetrical vibration along the line of centers, for the quantum change from 0 to 1 gives rise to a band at $4.25 \mu$. For the quantum changes 1 to 2 , the band falls at $4.30 \mu$, for 2 to 3 at $4.34 \mu$, and for 3 to 4 at $4.38 \mu$. In emission the quantum numbers change from large to small, but the energy changes are the same between the two states for absorption or emission. It is not possible to observe distinct bands corresponding to the different quantum jumps in the 4- to 5- $\mu$ region, as there is considerable overlapping of the various states. Also the quantum states of the other vibrations vary and produce a slight shift in the bands. The temperature measured from the inner cone to the outer surface of the flame varies considerably.

In addition, there is a change in the intensities of rotational lines as the temperature is increased. With all these effects on the energy emitted, it would be difficult to give a good approximation of the relative number of molecules represented in each state. The radiation of this band can be observed to about $5.0 \mu$, and this indicates that there is a considerable number of molecules in the higher rotational and vibrational states. The two bands, observed at 2.69 and $2.72 \mu$, arise from the combinations $\nu_{3}+\nu_{1}$, and $\nu_{3}+2 \nu_{2}$. In absorption, three bands occur that arise from the combinations $\nu_{3}+4 \nu_{2}, 2 \nu_{2}+\nu_{1}+\nu_{3}$, and $2 \nu_{1}+\nu_{3}$, whose wavelengths are $2.05,2.01$, and $1.96 \mu$, respectively. All three bands are of the doublet type and do not have the strong zero branch that would make it possible to separate them. These three bands probably produce the one maximum that is observed at $1.95 \mu$

In the $15-\mu$ region, the various states give rise to absorption bands with a zero branch. Therefore, the separate bands produced by different quantum 
jumps are discernible. For example, the band at $13.88 \mu$ is produced by the change $\left(10^{\circ} 0\right)$ to the $\left(01^{1} 0\right)$, that is $\nu_{1}-\nu_{2}$. The appearance of this band with such a high relative intensity indicates that the energy of the inactive frequency $\nu_{1}$, is transferred to the $\nu_{2}$ level, where it can be radiated. The possibility of a transfer of the energy from one of the higher states of the inactive frequency to a state of an active frequency has been suggested by Gaydon [8].

The strong maximum shown in figure 7 probably arises from the transitions $\left(02^{2} 0\right)$ to $\left(01^{1} 0\right)$ and $\left(01^{1} 0\right)$ to $\left(00^{0} 0\right)$. Also the transitions $\left(03^{1} 0\right)$ to $\left(02^{2} 0\right)$ at $15.46 \mu,\left(02^{0} 0\right)$ to $\left(01^{1} 0\right)$ at $16.18 \mu$, and $\left(03^{1} 0\right)$ to $\left(02^{2} 0\right)$ at $16.75 \mu$, should appear in this region. There is some indication of these bands in the observations made with the grating spectrometer, but this region should be restudied to definitely establish the presence of these transitions.

The intensity of the bands of $\mathrm{CO}_{2}$ in emission is different from the intensity in absorption. The probability coefficients of the population of states are well known and vary as the third power of the frequency in emission, although in absorption these coefficients are independent of frequency. That is, two absorption bands of equal intensity and having frequencies of $1,000 \mathrm{~cm}^{-1}$ and 2,000 $\mathrm{cm}^{-1}$, respectively, would have an intensity ratio of 1 to 8 in emission. Shaefer and Phillips [9] state that the $4.25-\mu$ band is about 28 times as strong as the $2.75-\mu$ band in the absorption spectrum of $\mathrm{CO}_{2}$. In the Bunsen flame the ratio is about 4 to 1 for the emitted energy of these two bands. However, when the factor $(3700 / 2300)^{3}$ is multiplied by 4 , a value close to 17 is obtained. It is difficult to make accurate intensity measurements, and the values given above are rough or approximate figures. The check is sufficiently close to suggest that the same type of excitation is present in each band, and that it is not necessary to assume a chemical reaction rather than thermal excitation. However, the approximations made in this calculation are not accurate and the possibility of a combination of thermal and other excitations cannot be ruled out

When the long wavelength band at $665 \mathrm{~cm}^{-1}$ is considered, there is a large decrease in the energy emitted because of the dependence on the third power of the frequency. The ratio of intensities in absorption must be multiplied by $(2300 / 665)^{3}$ to obtain the ratio of intensities in emission. This gives a value of approximately 120 to 1 for the intensity ratio of the $4.36-\mu$ band to $15.0-\mu$ band. For a more accurate determination other factors would have to be taken in consideration, but as the intensity measurements are not very accurate small correction terms would not be significant. The value of 120 to 1 for the ratio of intensities of these bands probably explains why Garner and Johnson [5] did not observe this band as they stated that an intensity ratio of only 50 to 1 was necessary for detection. It should be emphasized that in calculating these intensities only rough approximations have been used, and a much more refined mathematical treatment, taking into consideration the various energy states involved, as well as other factors, is necessary before an accurate value can be arrived at. However, such estimates are valuable in predicting the order of magnitude to be expected in the intensity measurements.

In figure 6 the deflection as shown in the broken curve is the amount of energy emitted after making the correction for absorption of $\mathrm{CO}_{2}$ in the air. This deflection is about equal to the deflection obtained in the $4.36-\mu$ band. After taking into consideration the different dispersions of the $\mathrm{NaCl}$ and $\mathrm{KBr}$ prisms, and the different slit widths used in the two regions, the ratio of the observed energies in emission for the two bands is found to be roughly 100 to 1 . This is approximately the same value as found in the calculated intensity ratio for emission. This suggests the possibility that this band is excited by the same mechanism as the $4.36-\mu$ band. However, because of the roughness of the approximations, this calculation cannot be taken as proof of the nature of the energy.

Gaydon [8] calculated the radiative life, or time of transition, from one state to another from observed absorption measurements and found that for the $\nu_{3}$ vibration this value is about 0.01 second, and that $\nu_{2}$ has a time of transition of about 0.2 second. Because of the greater transition life of $\nu_{2}$, he concludes that most of the energy of the $14.97-\mu$ band is lost by collisions and does not enter into the radiation process. Gaydon further states that the failure to observe this band is proof that the emission is produced by chemical rather than thermal causes. If the excitation is thermal, the band should be present and have an intensity in accordance with theoretical calcula- 
tions, because the population of the excited states would be increased and decreased by about the same amount in thermal exchanges. That thermal exchange could be the means of the energy radiation has been shown in the discussion of the ratio of the observed intensities of the $\nu_{2}$ and the $\nu_{3}$ vibrations. Gaydon has a very complete and interesting discussion in his book in regard to the combustion process and the influence of $\mathrm{H}_{2} \mathrm{O}$ and other substances on the energy emitted by the flame.

The emission spectrum of water vapor consists of vibrational-rotational bands and pure rotational lines. The two $\mathrm{OH}$ vibrations in the water vapor molecule give rise to bands at 2.66 and 2.74 in absorption. In figure 2 the water band is well separated from the $2.7-\mu$ region and has its maximum energy at $2.49 \mu$. There is an additional maximum on the short wavelength side of this band, but it is not well resolved. The change in frequency of the $\mathrm{H}_{2} \mathrm{O}$ molecule in emission as compared to the corresponding frequency in absorption may be brought about by the molecule being in the higher rotational states. Randall, Dennison, Ginsburg, and Weber [10] have shown in accounting for the rotational lines in the absorption spectrum of water vapor that, for the higher energy states, there is a change in the angle of the water molecule. They state that the bond angle of the molecule changes from its equilibrium value of $104^{\circ} 36^{\prime}$ to $98^{\circ} 52^{\prime}$, and the $\mathrm{OH}$ distance changes from 0.9558 to 0.9640 A when $J=11$. Therefore, if the molecule at the temperature of emission is in the higher rotational states, it would not have the same spread between the maxima in the $P$ and $R$ branches as observed in absorption. The combination band $\nu_{3}+\nu_{2}$ is observed at $1.81 \mu$. This band corresponds to the band at $1.89 \mu$ in absorption.

It is not possible to identify the exact location of the $6.26-\mu$ band, but it is shifted to about $6.35 \mu$. By subtracting $\nu_{3}$ from $\nu_{3}+\nu_{2}$, a value of $1508 \mathrm{~cm}^{-1}$ or about $6.63 \mu$ is obtained, which corresponds approximately to the observed value. The $6.26-\mu$ absorption band of $\mathrm{H}_{2} \mathrm{O}$ has a greater intensity than the 2.66- $\mu$ band. However, in emission the $2.49-\mu$ band is more intense than the $6.35-\mu$ band. The change in intensity ratio between absorption and emission is explained by the third power of frequency that enters into the probability coefficients of emission. This effect has been discussed in connection with the intensities of the $\mathrm{CO}_{2}$ emission bands and will not be repeated for the $\mathrm{H}_{2} \mathrm{O}$ bands.

There are several small bands of $\mathrm{CO}_{2}$ and $\mathrm{H}_{2} \mathrm{O}$ in the region below $1.8 \mu$, but they have not been studied in detail and no attempt has been made to classify them. In figure 5 it is seen that the rotational-vibrational band of water vapor becomes very weak in the spectral region of $9 \mu$. In this region it is difficult to classify a line as part of the rotational-vibrational band or as a pure rotational line. The lines increase in intensity from 9 to $15 \mu$. The resolution is good with a $\mathrm{NaCl}$ prism at 12 and $13 \mu$, and much more detail of the lines can be seen than with a $\mathrm{KBr}$ prism. However, beyond $14 \mu$ the $\mathrm{KBr}$ prism is more suitable. Some of the stronger emission lines have been compared with the absorption lines for the same spectral region, and it is found that the lines in emission do not appear at the same frequency as the absorption lines. This variation in frequency is probably caused by the emission lines being produced by transitions when the molecule occupies higher states, and the absorption lines being produced by lower energy transitions. These emission lines should be measured on a grating instrument. Then it may be possible to classify properly the emission lines, as has been done for rotational lines in absorption.

In figure 2 there are shown 15 rotational lines in the region between 2.8 and $3.2 \mu$. Some lines are also present below $2.8 \mu$, but because of the absorption of the water vapor in the air and the $\mathrm{CO}_{2}$ emission band, the lines below $2.8 \mu$ could not be measured accurately. The fifteen lines observed are spaced about $22 \mathrm{~cm}^{-1}$ apart. With higher resolution the band might show additional fine structure. These rotational lines, because of the large frequency spacing, evidently involve hydrogen. The $\mathrm{OH}$ band for many substances has been observed in the region of $2.8 \mu$. The moment of inertia of the $\mathrm{OH}$ group is such that a spacing somewhat different than that observed in these lines would be expected. The observed spacing of these rotational lines corresponds more clsely to the spacing calculated for the $\mathrm{H}_{2} \mathrm{O}$ molecule when the quantum changes are $\Delta k=0$ and $\Delta j=-1$. These lines correspond to the changes from $j=10$ to $j=24$. In the flame of burning hydrogen these lines are much more intense, and several additional lines have been 
observed. These data will be presented in a subsequent article.

A small emission band has been observed in the region of $3.32 \mu$. This band is about 12 $\mathrm{cm}^{-1}$ less in frequency than the methane band. The intensity of the band is very low. The region of maximum energy has the appearance of a zero branch. As this is the part of the infrared spectrum, where characteristic vibrations of $\mathrm{CH}$ are found in absorption, this band is classified as being produced by the $\mathrm{CH}$ bond. As no rotational structure has been observed, it is not possible to distinguish between $\mathrm{CH}, \mathrm{CH}_{2}, \mathrm{CH}_{3}$, or $\mathrm{CH}_{4}$, and this band may arise from any one of these atomic groups.

Because of the strong emission of the $\mathrm{CO}_{2}$ band in the region of $4.64 \mu$, no radiation that could be attributed to $\mathrm{CO}$ would be discerned unless it was of considerable intensity. As no bulge was observed, any energy that the CO molecule radiates in this region must be of low intensity. In the region of $2.3 \mu$, the flame is almost void of radiation, hence a low-intensity band due to the harmonic of $\mathrm{CO}$ could have been detected if it occurred. This leads to the conclusion that $\mathrm{CO}$ contributes very little to the total emission of the Bunsen burner.

The absence of a band in the region of $2.2 \mu$ indicates that there is not a first harmonic of the $\nu_{3}$ vibration of $\mathrm{CO}_{2}$. This is in agreement with the selection rules that apply to absorption.

\section{Conclusion}

In this work the infrared emission of a Bunsen flame has been measured from 1.8 to $24 \mu$ and good resolution, obtained by a LiF prism, has been employed for the study of the region from
1.8 to $3.6 \mu$. Many bands have been resolved that have not been reported previously by other observers. It appears that a study of the infrared radiation with higher resolution than that used in this work would result in showing more detail that would be of great value in the formulation of a theory of combustion. This would also allow the more accurate calculation of the energy states. In addition to the need for better resolution, well-regulated sources should be used so that the various gases and the oxygen or air may be varied in known amounts. A vacuum spectrometer or one in which the air about it is free from $\mathrm{CO}_{2}$ and water vapor would allow more accurate measurements in several regions of the spectrum.

\section{References}

[1] J. W. Ellis, Phys. Rev. 26, 469 (1925); C. R. Bailey and K. H. Lih, Trans. Faraday Soc. 25 (1929).

[2] V. Kondratjew, Z. Physik 63, 322 (1930) ; V. Kondratjew and M. Ziskin, Acta Physicochim. (USSR) 7, 65 (1937); W. M. Vaidya, Proc. Roy. Soc. [A] 14\%, 513 (1934) ; A. G. Gaydon, Proc. Roy. Soc. [A] 179, 439 (1942).

[3] E. F. Barker, Astrophys. J. 55, 391 (1922).

[4] H. Rubens and E. Aschkinass, Ann. Physik u. Chem. 64, 584 (1898).

[5] W. E. Garner and C. H. Johnson, Phil. Mag. [7], 3, 97 (1927).

[6] A. Adel and D. M. Dennison, Phys. Rev. 43, 716 (1933).

[7] G. Herzberg, Infrared and Raman spectra of polyatomic molecules (D. Van Nostrand \& Co., Inc., New York, N. Y., 1945).

[8] A. G. Gaydon, Spectroscopy and combustion theory (Chapman \& Hill, Ltd., London, 1942).

[9] C. Shaefer and B. Phillips, Z. Physik 36, 641 (1926).

[10] H. M. Randall, D. M. Dennison, N. Ginsburg, and L. R. Weber, Phys. Rev. 52, 160 (1937).

Washington, November 21, 1947. 